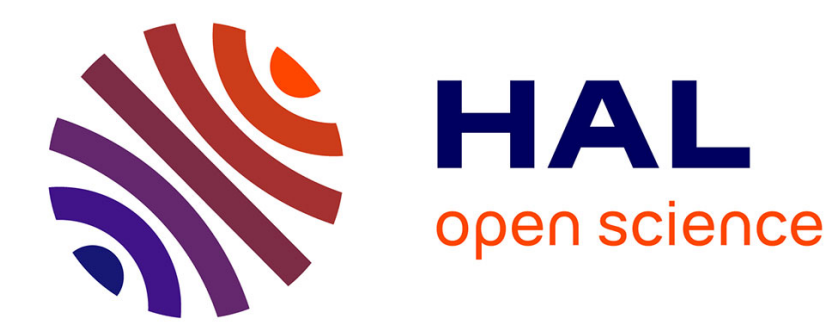

\title{
MÜNTZ SPACES AND SPECIAL BLOCH TYPE INEQUALITIES
}

\author{
Pascal Lefèvre
}

\section{To cite this version:}

Pascal Lefèvre. MÜNTZ SPACES AND SPECIAL BLOCH TYPE INEQUALITIES. 2017. hal01642143

\section{HAL Id: hal-01642143 \\ https://hal.science/hal-01642143}

Preprint submitted on 21 Nov 2017

HAL is a multi-disciplinary open access archive for the deposit and dissemination of scientific research documents, whether they are published or not. The documents may come from teaching and research institutions in France or abroad, or from public or private research centers.
L'archive ouverte pluridisciplinaire HAL, est destinée au dépôt et à la diffusion de documents scientifiques de niveau recherche, publiés ou non, émanant des établissements d'enseignement et de recherche français ou étrangers, des laboratoires publics ou privés. 


\title{
MÜNTZ SPACES AND SPECIAL BLOCH TYPE INEQUALITIES
}

\author{
PASCAL LEFÈVRE
}

\begin{abstract}
In this paper, we are interested in classical Müntz spaces and their properties. We first reprove Müntz theorem and obtain the Clarkson-Erdös-Schwartz expansion in the non dense case for some general abstract Müntz spaces without invoking any blow up property, but using classical complex variables tools. We introduce new Bloch type Müntz spaces and investigate the validity of Bloch type inequalities in this framework.
\end{abstract}

\section{INTRODUCTION}

The Müntz theorem (see [17] for the original result and [2] for a good recent survey) gives an answer to a very natural question on the extension of the Weierstrass theorem in approximation theory in the framework of $L^{p}(0,1)$ and $C([0,1])$. It is a natural question to understand the geometry (and the behavior of their operators) of the Banach spaces spanned by monomials in the non dense case: that is the purpose of the monograph [10], and a series of recent results [1], [5], [6], [7], [16] or [18].

More precisely, in the sequel, we shall use functions $\mathrm{e}_{\lambda}: t \in(0,1) \mapsto t^{\lambda}$ for $\lambda \in \mathbb{R}^{+}$. Given $\Lambda \subset \mathbb{R}^{+}$, we denote by $M_{\Lambda}$, the vector space spanned by the functions $\mathrm{e}_{\lambda}$ where $\lambda \in \Lambda$. For $p \geq 1$, we denote by $M_{\Lambda}^{p}$ (resp. $\left.M_{\Lambda}^{\infty}\right)$ the closure of $M_{\Lambda}$ in $L^{p}(0,1)$ (resp. in $C([0,1])$ ), equipped with their usual norm. When $\Lambda=\left\{\lambda_{n}\right\}$ (concretely we shall always work with an increasing sequence in the sequel) satisfies the

$$
\text { Müntz condition } \quad \sum_{\lambda_{n} \neq 0} \frac{1}{\lambda_{n}}<\infty
$$

then we get a strict subspace of $L^{p}(0,1)$ and $C([0,1])$ : this is the conclusion of the classical Müntz theorem.

Let us emphasize that, as soon as we restrict to the case $\Lambda \subset \mathbb{N}$, the functions in the space spanned by the $\mathrm{e}_{\lambda}$ are "true" (algebraic) polynomials. In this case, there are already many open questions on the structure of the spaces $M_{\Lambda}^{p}$, which turns out to be some spaces of analytic functions on the complex unit disk. Indeed the Clarkson-Erdös-Schwartz theorem (see [10] for instance, and section 2 below) states that, up to the gap condition (see (GC) below), functions of Müntz spaces $M_{\Lambda}^{p}$ (resp. $\left.M_{\Lambda}^{\infty}\right)$ are precisely functions $f \in L^{p}(0,1)$ (resp. in $C([0,1])$ ) which are analytic on $(0,1)$ with an expansion

$$
f(t)=\sum a_{n} t^{\lambda_{n}} \quad \text { for almost every } t \in[0,1) .
$$

Up to a change of our original function on a set with measure 0 , we shall assume in sections 3 and 4 that this expansion is valid on $[0,1)$. In particular our functions have derivatives on $[0,1)$.

2010 Mathematics Subject Classification. 30B10.

Key words and phrases. Müntz spaces, Power series, lacunary sequences, Bloch type inequalities. 
Let us give more details on the framework. We are going to work mainly (at least in the three first sections) with some "classical" functions spaces over $(0,1)$, that is Banach spaces $X$ of functions on $(0,1)$ with the following properties

(i) $C_{0}([0,1])=\{f \in C([0,1]) \mid f(0)=0\} \subset X$ continuously: there exists $k>0$ such that for every $f \in C_{0}([0,1])$,

$$
\|f\|_{X} \leq k\|f\|_{\infty}
$$

(ii) $C_{0}([0,1])$ is dense in $X$.

Keep mainly in mind the examples of spaces $C_{0}([0,1])$ and $L^{p}(0,1)=L^{p}([0,1], m)$, where $m$ is the Lebesgue measure. In another direction, we shall introduce some Bloch type spaces in section 4 and we shall see that the Clarkson-Erdös-Schwartz expansion holds too in this framework under the Müntz condition, although (i) and (ii) do not hold.

In section 2, we are going to recover Müntz theorem and Clarkson-Erdös-Schwartz for rather general Banach spaces of functions on $[0,1)$, containing polynomials. The gap condition

$$
\inf \left(\lambda_{n+1}-\lambda_{n}\right)>0
$$

will be sometimes involved.

With our approach based on Blaschke products, we do not need to invoke any blow up property to get Müntz theorem and Clarkson-Erdös-Schwartz expansion for classical spaces. We shall say that $X$ has the blow up property if the blow up operator $T_{\rho}: f \in X \longmapsto f(\rho \cdot) \in X$ is bounded for every $\rho \in(0,1)$ and

$$
\sup _{\rho_{0}<\rho<1}\left\|T_{\rho}\right\|_{X}<\infty \quad \text { for any } \rho_{0} \in(0,1)
$$

Exploiting this property turns out to be very efficient to deduce from the case $X=C_{0}([0,1])$ Müntz and Clarkson-Erdös-Schwartz theorems for more general spaces, using a duality argument, but also some complementary technical tools (see [10]). In the present paper, we use a more direct argument to get the main results for classical spaces. We only use partially the blow up property in the context of weighted Bloch type spaces with large parameter (see the proof of Th.4.2 below), but avoiding the requirement of more machinery.

In section 3, we focus on the Gurariy-Macaev theorem. We point out that this nice result implies that the Bergman norm and the $L^{p}(0,1)$-norm are equivalent on the space of lacunary Taylor series. We also revisit an almost isometric version of this theorem in the hilbertian framework and give a very elementary proof of this version.

In section 4, we focus on Bloch type inequalities for Müntz spaces. We first introduce a Bloch type space of function on $(0,1)$ and prove a version of the ClarksonErdös-Schwartz theorem in this framework. We compare this space to classical Müntz spaces $M_{\Lambda}^{p}$, equivalently we consider special inequalities between the $L^{p}$ norm of a Müntz polynomial and a weighted norm of its derivative. Such inequalities hold in the lacunary case but we shall construct some counterexamples showing that they do not hold in general for a given $\Lambda$. We also show that for some $\Lambda \subset \mathbb{N}$, there exists some function in the associated Müntz space $M_{\Lambda}^{\infty}$ such that the derivative does not belong to the weak $L^{1}$ space over $(0,1)$.

We did no try to adopt the most general point of view. For instance in the $L^{p}$ framework, we could consider $\mathrm{e}_{\lambda}$ when $\lambda>-\frac{1}{p}$ (or even complex exponent) and 
some phenomenom when $\lambda$ is close to $-\frac{1}{p}$ are similar when $\lambda \rightarrow \infty$. Actually, as already mentioned, many natural and interesting questions are still completely open when $\Lambda \subset \mathbb{N}$.

In the sequel, we shall sometimes use the notation $A \lesssim B$, which means that there exists $k>0$ such that $A \leq k B$ where $k$ depends maybe on $p$ (or on $\Lambda$ ). In the same way, $A \approx B$ means that $A \lesssim B$ and $B \lesssim A$.

\section{Müntz theorem and Clarkson-Erdös-Schwartz Theorem}

In this section, we are interested in proving in our framework two classical results of the theory of Müntz spaces. The spirit and the tools of the following proofs are not really new, nevertheless it is slightly different from the usual presentation, as chosen in [10] for instance, where the proof uses Cauchy determinant whereas we use a Blaschke product. The spirit of the proof is partially inspired from part of the proof of the Müntz theorem given in [19] in the special case $X=C([0,1])$ (see $[4$, E.13, p.192] too). We push this idea farther and adapt it to get both Müntz Theorem and Clarkson-Erdös-Schwartz expansion for Banach spaces of functions on $(0,1)$ whose norm dominates the $L^{1}$ norm. As far as we know, this strategy is new and our approach relies on the following proposition.

Proposition 2.1. Assume that $\sum \frac{1}{\lambda_{n}}<+\infty$.

- For every $m>-1$, we have $d_{L^{1}}\left(\mathrm{e}_{m}, \operatorname{span}\left\{\mathrm{e}_{\lambda} ; \lambda \in \Lambda \backslash\{m\}\right\}\right)>0$.

- When (GC) is fulfilled, there exists a function $\gamma: \Lambda \rightarrow \mathbb{R}^{+}$satisfying $\gamma(m) \rightarrow 0$ (when $m \rightarrow+\infty$ ) and

$$
\forall m \in \Lambda, \quad d_{L^{1}}\left(\mathrm{e}_{m}, \operatorname{span}\left\{\mathrm{e}_{\lambda} ; \lambda \in \Lambda \backslash\{m\}\right\}\right) \geq \mathrm{e}^{-(m+1) \gamma(m)} .
$$

Here $d_{L^{1}}(f, A)=\inf \left\{\|f-a\|_{1} ; a \in A\right\}$ where $f \in L^{1}(0,1)$ and $A \subset L^{1}(0,1)$.

Proof. In the sequel for any $a \in \mathbb{R}$, we denote $\mathbb{C}_{a}=\{z \in \mathbb{C} ; \operatorname{Re}(z)>a\}$.

The Blaschke product of the right half plane

$$
B(z)=\prod_{n=0}^{\infty} \frac{\lambda_{n}+1-z}{\lambda_{n}+1+z}
$$

is defined for every $z \in \mathbb{C}_{-1}$ and $B$ is holomorphic on $\mathbb{C}_{-1}$. Moreover $B$ is inner: for every $z \in \overline{\mathbb{C}_{0}}$, we have $|B(z)| \leq 1$.

Therefore, the function $F(z)=\frac{2}{(1+z)^{2}} B(z)$ defines an holomorphic function on $\mathbb{C}_{-1}$ such that $s \in \mathbb{R} \longmapsto \widetilde{F}(s)=F(i s)$ belongs to $L^{1}(\mathbb{R})$, with norm less than $2 \pi$. Thanks to the Cauchy formula, we have

$$
\forall z \in \mathbb{C}_{0}, \quad F(z)=\frac{1}{2 \pi} \int_{-\infty}^{+\infty} \frac{\widetilde{F}(s)}{z-i s} d s
$$

and the function

$$
\mu_{\Lambda}(x)=\frac{1}{2 \pi} \int_{-\infty}^{+\infty} \widetilde{F}(s) x^{-i s} d s \quad \text { for } x \in[0,1]
$$

defines a continuous function with $\left\|\mu_{\Lambda}\right\|_{\infty} \leq 1$. The continuity at point 0 follows from the Riemann-Lebesgue lemma, since actually $\mu_{\Lambda}$ is the Fourier transform of $\widetilde{F}$ at point $\ln (x)$. 
The crucial point is the property

$$
\forall z \in \mathbb{C}_{0}, \quad \int_{0}^{1} x^{z-1} \mu_{\Lambda}(x) d x=F(z)=\frac{2}{(1+z)^{2}} \prod_{n=0}^{\infty} \frac{\lambda_{n}+1-z}{\lambda_{n}+1+z},
$$

which follows from Fubini's theorem. Indeed, for every $z \in \mathbb{C}_{0}$, we have

$$
\int_{0}^{1} x^{z-1} \mu_{\Lambda}(x) d x=\frac{1}{2 \pi} \int_{-\infty}^{+\infty}\left(\int_{0}^{1} x^{z-1} x^{-i s} d x\right) \widetilde{F}(s) d s=\frac{1}{2 \pi} \int_{-\infty}^{+\infty} \frac{1}{z-i s} \widetilde{F}(s) d s
$$

and (3) follows now from (1) and the definition of $F$.

Applying (3) with $z=m+1$ where $m \in \mathbb{C}_{-1}$, we get

$$
\text { for every } m \in \mathbb{C}_{-1}, \quad \int_{0}^{1} x^{m} \mu_{\Lambda}(x) d x=\frac{2}{(m+2)^{2}} \prod_{n=0}^{\infty} \frac{\lambda_{n}-m}{\lambda_{n}+m+2}
$$

which vanishes if and only $m \in \Lambda$.

Now, consider $\Lambda_{m}=\Lambda \backslash\{m\}$. For every polynomial $P \in \operatorname{span}\left\{\mathrm{e}_{\lambda} ; \lambda \in \Lambda_{m}\right\}$, we have, thanks to (4),

$$
\int_{0}^{1}\left(x^{m}-P(x)\right) \mu_{\Lambda_{m}}(x) d x=\int_{0}^{1} x^{m} \mu_{\Lambda_{m}}(x) d x=\frac{2}{(m+2)^{2}} \prod_{\substack{n \geq 0 \\ \lambda_{n} \neq m}} \frac{\lambda_{n}-m}{\lambda_{n}+m+2} .
$$

A fortiori, for every real number $m>-1$,

$$
\left\|\mathrm{e}_{m}-P\right\|_{1} \geq\left\|\mathrm{e}_{m}-P\right\|_{1} \cdot\left\|\mu_{\Lambda_{m}}\right\|_{\infty} \geq \frac{2}{(m+2)^{2}} \prod_{\substack{n \geq 0 \\ \lambda_{n} \neq m}} \frac{\left|\lambda_{n}-m\right|}{\lambda_{n}+m+2}>0
$$

and the first part of the proposition is proved.

Now, we assume that the gap condition is fulfilled: $\inf \left(\lambda_{n+1}-\lambda_{n}\right) \geq \delta$ for some $\delta \in(0,1)$. We focus on (5) when $m \in \Lambda$ and aim to get a lower bound estimate of the right hand side. We split the product into three part and estimate each of them.

We first easily check that

$$
\prod_{\substack{n \geq 0 \\ \lambda_{n}>2 m}} \frac{\lambda_{n}-m}{\lambda_{n}+m+2}=\exp \left(-\sum_{\lambda_{n}>2 m} \ln \left(1+\frac{2(m+1)}{\lambda_{n}-m}\right)\right) \geq \exp \left(-4(m+1) \rho_{2 m}(\Lambda)\right)
$$

where $\rho_{s}(\Lambda)$ stands for $\sum_{\lambda_{n}>s} \frac{1}{\lambda_{n}}$.

Denote $N_{s}=\left|\left\{n \in \mathbb{N} ; \lambda_{n} \leq s\right\}\right|$. We have $N_{s}=o(s)$ (when $s \rightarrow+\infty$ ) because $\sum 1 / \lambda_{n}<+\infty$.

Let $n$ be an integer such that $m<\lambda_{n} \leq 2 m$. We point out that $\lambda_{n}-m \geq$ $\delta(n-d)$ when $m=\lambda_{d}$. We get this way distincts integers $n-d$ running over $\left\{1, \ldots, N_{2 m}-N_{m}\right\}$.

Using the inequality $k ! \geq k^{k} \mathrm{e}^{-k}$ (which is valid for any integer $k \geq 1$ ), we obtain

$$
\begin{aligned}
\prod_{m<\lambda_{n} \leq 2 m} \frac{\lambda_{n}-m}{\lambda_{n}+m+2} & \geq \frac{\left(N_{2 m}-N_{m}\right) ! \delta^{N_{2 m}-N_{m}}}{(3 m+2)^{N_{2 m}-N_{m}}} \\
& \geq \delta^{N_{2 m}-N_{m}} \exp \left(-(m+1) \theta\left(\left(N_{2 m}-N_{m}\right) /(m+1)\right)\right)
\end{aligned}
$$


where the function $\theta$ is defined by $\theta(t)=t \ln (3 \mathrm{e} / t)$ when $t>0$ and $\theta(0)=0$.

In the same way, we have

$$
\prod_{\substack{n \geq 0 \\ m>\lambda_{n}}} \frac{m-\lambda_{n}}{\lambda_{n}+m+2} \geq \delta^{N_{m}} \exp \left(-(m+1) \theta\left(\left(N_{m}-1\right) /(m+1)\right)\right)
$$

We define

$\gamma(m)=\frac{-\ln (2)+2 \ln (m+2)}{m+1}+4 \rho_{2 m}(\Lambda)-\frac{N_{2 m} \ln (\delta)}{m+1}+\theta\left(\frac{N_{2 m}-N_{m}}{(m+1)}\right)+\theta\left(\frac{N_{m}-1}{(m+1)}\right)$

and clearly $\gamma(m) \longrightarrow 0$ when $m \rightarrow \infty$ and the conclusion follows.

As an immediate corollary, we recover the non dense part of the Müntz theorem for some general classes of Banach spaces.

Theorem 2.2. [abstract Müntz theorem] Let $X$ be a Banach space of functions on $(0,1)$ such that the following inequalities hold continuously: $C_{0}([0,1]) \subset X \subset L^{1}(0,1)$ and such that $C_{0}([0,1])$ is dense in $X$. Let $\Lambda=\left\{\lambda_{n}\right\}$ be an increasing sequence of positive numbers.

Then span $\left\{\mathrm{e}_{\lambda_{n}}\right\}$ is dense in $X$ if and only if $\sum \frac{1}{\lambda_{n}}=+\infty$.

Moreover, when the series converges, for every $m \notin \Lambda$, we have $\mathrm{e}_{m} \notin \overline{\operatorname{span}\left\{\mathrm{e}_{\lambda_{n}}\right\}}$

Let us mention that the space $C([0,1])$ does not fulfill all the hypothesis of this theorem. Nevertheless, it suffices to include the constant functions (i.e. to add $\mathrm{e}_{0}$ in the span) since $C([0,1])=\mathbb{C e}_{0}+C_{0}([0,1])$.

Proof. Assume that $\sum 1 / \lambda_{n}<+\infty$, then Prop.2.1 implies that for every $m$ not belonging to $\Lambda$, the function $\mathrm{e}_{m}$ does not belong to $M_{\Lambda}^{1}$. It implies that $\mathrm{e}_{m}$ does not belong to $M_{\Lambda}^{X}$ neither, which is a strong negation of the density.

Now assume that $\sum 1 / \lambda_{n}=+\infty$, then we know that $\operatorname{span}\left\{\mathrm{e}_{\lambda_{n}}\right\}$ is dense in $C_{0}([0,1])$ by the classical version of the Müntz theorem, but for sake of completeness let us reproduce here the (now classical) argument from [19]: assume that a measure $\mu$ on $(0,1)$ satisfies

$$
\Phi(z)=\int_{(0,1)} t^{z} d \mu(t)=0 \quad \text { for every } z \in \Lambda .
$$

It means that the function $\Phi$ which is actually defined, bounded and holomorphic on the right half plane $\mathbb{C}_{0}$, vanishes on $\Lambda$. Therefore, $\Phi$ belongs to the space $H^{\infty}\left(\mathbb{C}_{0}\right)$ (the Hardy space $H^{\infty}$ of the right half plane) and the condition $\sum 1 / \lambda_{n}=+\infty$ means that the Blaschke condition on zeroes of non trivial such functions is not satisfied, hence $\Phi=0$ and a fortiori $\Phi$ actually vanishes on positive integers. The Weierstrass theorem implies that $\mu=0$ and $\operatorname{span}\left\{\mathrm{e}_{\lambda_{n}}\right\}$ is dense in $C_{0}([0,1])$. Since $C_{0}([0,1])$ is dense in $X$, the "if" part is proved.

We are going to deduce too the Clarkson-Erdös-Schwartz Theorem from Prop.2.1. We first need

Lemma 2.3. Let $\Lambda$ satisfying the gap condition (GC) and $\sum 1 / \lambda_{n}<+\infty$. For every $\varepsilon>0$, there exists $C_{\varepsilon}>0$ (depending on $\Lambda$ ) satisfying:

For every $P \in \operatorname{span}\left\{\mathrm{e}_{\lambda_{n}}\right\}$, written $P=\sum a_{n} \mathrm{e}_{\lambda_{n}}$, we have

$$
\text { for every } n, \quad\left|a_{n}\right| \leq C_{\varepsilon}(1+\varepsilon)^{\lambda_{n}}\|P\|_{1} \text {. }
$$


In particular, the linear map $\mathrm{e}_{\lambda}^{*}: P \in\left(\operatorname{span}\left\{\mathrm{e}_{\lambda_{n}}\right\},\|\cdot\|_{1}\right) \longmapsto a_{\lambda} \in \mathbb{C}$ (where $\left.P=\sum a_{\lambda} \mathrm{e}_{\lambda}\right)$ is continuous, hence extends to a (bounded) linear functional on $M_{\Lambda}^{1}$. We shall still denote $\mathrm{e}_{\lambda}^{*}$ this functional in the sequel.

Proof. Fix $m=\lambda_{n}\left(\right.$ where $\left.a_{n} \neq 0\right)$ and apply Prop.2.1 to $\tilde{P}=-a_{n}^{-1} \sum_{j \neq n} a_{j} \mathrm{e}_{\lambda_{j}}$. We get

$$
\left|a_{n}^{-1}\right|\|P\|_{1}=\left\|\mathrm{e}_{\lambda_{n}}-\tilde{P}\right\|_{1} \geq \mathrm{e}^{-\left(\lambda_{n}+1\right) \gamma\left(\lambda_{n}\right)} .
$$

We know that $\mathrm{e}^{\gamma\left(\lambda_{n}\right)}$ is bounded (by $C$ say) on one hand and is less than $1+\varepsilon$ for $n$ large enough (say $n \geq n_{\varepsilon}$ ) on the other hand. We denote

$$
C_{\varepsilon}=C \max \left\{1, \sup _{n \leq n_{\varepsilon}}\left(\frac{\mathrm{e}^{\gamma\left(\lambda_{n}\right)}}{1+\varepsilon}\right)^{\lambda_{n}}\right\}
$$

and we get the conclusion.

We are going to recover in the following statement the existence of a Taylor type expansion for usual spaces, without requiring the blow up property. This makes our proof of $(i)$ below different from the one in [10] and more direct.

Theorem 2.4 (Clarkson-Erdös-Schwartz). Let $\Lambda=\left\{\lambda_{n}\right\}$ be an increasing sequence of positive numbers satisfying the gap condition (GC) and $\sum 1 / \lambda_{n}<+\infty$.

Let $X$ be a Banach space of functions on $(0,1)$ such that the following inequality holds (continuously): $X \subset L^{1}(0,1)$. Let $f \in X$.

(i) $f \in M_{\Lambda}^{X} \Longrightarrow \exists\left(a_{n}\right)_{n} \in \mathbb{C}^{\mathbb{N}}, \quad f(t)=\sum_{n} a_{n} t^{\lambda_{n}} \quad$ for a.e. $t \in[0,1)$.

(ii) If moreover, $X$ satisfies (buc), $X \cap C([0,1])$ is dense in $X$ and $M_{\Lambda}^{\infty} \subset X$ (continuously) then

$$
\exists\left(a_{n}\right)_{n} \in \mathbb{C}^{\mathbb{N}}, \quad f(t)=\sum_{n} a_{n} t^{\lambda_{n}} \quad \text { for a.e. } t \in[0,1) \Longrightarrow f \in M_{\Lambda}^{X} .
$$

Proof. Thanks to Lemma 2.3, we know that the functionals $\mathrm{e}_{\lambda}^{*}$ are bounded on $M_{\Lambda}^{X}$ for every $\lambda \in \Lambda$ and that $\left\|\mathrm{e}_{\lambda}^{*}\right\| \leq C_{\varepsilon}(1+\varepsilon)^{\lambda}$ for every $\varepsilon \in(0,1)$.

Let us prove $(i)$. Assume that $f \in M_{\Lambda}^{X}$. By hypothesis, there exists $k>0$ (depending on $X$ only) such that $\|f\|_{1} \leq k\|f\|_{X}$.

Fix $x \in[0,1)$ and choose $\varepsilon$ such that $r=(1+\varepsilon) x \in[0,1)$, we have

$$
\left|\mathrm{e}_{\lambda_{n}}^{*}(f) x^{\lambda_{n}}\right| \leq C_{\varepsilon}(1+\varepsilon)^{\lambda_{n}} x^{\lambda_{n}}\|f\|_{1} \leq C_{\varepsilon}^{\prime}(1+\varepsilon)^{\lambda_{n}} x^{\lambda_{n}}\|f\|_{X}=C_{\varepsilon}^{\prime} r^{\lambda_{n}}\|f\|_{X}
$$

where $C_{\varepsilon}^{\prime}=k C_{\varepsilon}$, and we are then allowed to consider

$$
\widetilde{f}(x)=\sum_{n} \mathrm{e}_{\lambda_{n}}^{*}(f) x^{\lambda_{n}}, \text { where } x \in[0,1)
$$

There exists a sequence $\left(P_{j}\right)$ in $\operatorname{span}\left\{\mathrm{e}_{\lambda_{n}}\right\}$ converging to $f$ in $X$ by definition. We see that, for every $x \in[0,1)$, we have

$$
\left|P_{j}(x)-\tilde{f}(x)\right|=\left|\sum_{n}\left(\mathrm{e}_{\lambda_{n}}^{*}\left(P_{j}\right)-\mathrm{e}_{\lambda_{n}}^{*}(f)\right) x^{\lambda_{n}}\right| \leq C_{\varepsilon}^{\prime}\left(\sum_{n} r^{\lambda_{n}}\right)\left\|P_{j}-f\right\|_{X} .
$$

But the gap condition (GC) implies that $\sum_{n} r^{\lambda_{n}}<\infty$, since $\lambda_{n} \geq \lambda_{0}+\delta n$, where $\delta=\inf \left\{\lambda_{n+1}-\lambda_{n}\right\}$. We obtain that $\lim P_{j}(x)$ exists and is equal to $\widetilde{f}(x)$.

On the other hand, $\left(P_{j}\right)$ converges to $f$ in $L^{1}$ so Fatou's lemma implies that $\lim P_{j}=f$ a.e. We conclude that $f=\tilde{f}$ a.e. 
Let us prove $(i i)$. By hypothesis, there exists $k_{0}$ such that $k_{0}\|h\|_{\infty} \geq\|h\|_{X}$ for every $h \in M_{\Lambda}^{\infty}$.

Fix $\varepsilon>0$. We first claim that there exists some $\rho$ such that $\left\|T_{\rho}(f)-f\right\|_{X} \leq \varepsilon$. Indeed we know that $C=\sup _{\rho>1 / 2}\left\|T_{\rho}\right\|<\infty$ and that there exists some $g \in C([0,1])$ with $\|f-g\|_{X} \leq \varepsilon / 2(C+1)$. Therefore, for every $\rho>1 / 2$,

$$
\left\|T_{\rho}(f)-f\right\|_{X} \leq\left\|T_{\rho}(f-g)\right\|_{X}+\left\|T_{\rho}(g)-g\right\|_{X}+\|f-g\|_{X} \leq \frac{\varepsilon}{2}+\left\|T_{\rho}(g)-g\right\|_{X}
$$

and $\left\|T_{\rho}(g)-g\right\|_{X} \leq k_{0}\left\|T_{\rho}(g)-g\right\|_{\infty} \leq \varepsilon / 2$ if $\rho$ is close enough to 1 by uniform continuity of $g$. The claim is proved.

Since the Taylor series $\sum_{n} a_{n} X^{\lambda_{n}}$ has convergence radius at least $1, T_{\rho}(f)=f(\rho \cdot)$ belongs to $C([0,1])$ and there exists some $J$ such that

$$
\left\|T_{\rho}(f)-\sum_{n \leq J} a_{n} \rho^{\lambda_{n}} \mathrm{e}_{\lambda_{n}}\right\|_{\infty} \leq \frac{\varepsilon}{k_{0}} .
$$

We conclude that $\left\|f-\sum_{n \leq J} a_{n} \rho^{\lambda_{n}} \mathrm{e}_{\lambda_{n}}\right\|_{X} \leq 2 \varepsilon$.

\section{Around the Gurariy-Macaev theorem}

An important consequence of the Clarkson-Erdös-Schwartz theorem is that a Müntz space associated to an increasing sequence $\Lambda$ is a space of analytic functions on $(0,1)$. When we focus on the particular case of a set of integers $\Lambda$, it can also be seen as a space of analytic functions on the open unit disk $\mathbb{D}$. Having in mind classical Hardy spaces $H^{p}$ and Bergman spaces $\mathcal{A}^{p}$, it is natural to wonder whether they are comparable to Müntz spaces, and (more precisely) compare the $L^{p}$ norm, the norm on $H^{p}$ and the norm on $\mathcal{A}^{p}$ of lacunary polynomials. Consider the $L^{p}$ norm and take a lacunary sequence (of integers, to guarantee the analycity), the Gurariy-Macaev theorem (see below) shows that the norm of a linear combination of the $\mathrm{e}_{\lambda}$ behaves like a weighted $\ell^{p}$ norm of the coefficient, whereas in $H^{p}$ the norm of a linear combination of the $z^{\lambda}$ behaves like the $\ell^{2}$ norm of the coefficients for the Hardy space $H^{p}$. Concerning the Bergman norm of such a linear combination, we shall see below that it behaves like its $L^{p}$ norm.

In order to make more precise these remarks in the sequel, we need and recall first the following theorem due to Gurariy-Macaev [11]. The full statement involves the space $c$ of convergent sequences (equipped with the usual sup norm $\|\cdot\|_{\infty}$ ). This space is isomorphic to the space $c_{0}$ of null sequences.

Theorem 3.1 (Gurariy-Macaev). Let $p \geq 1$. TFAE

- The normalized sequence $\left(\left(p \lambda_{n}+1\right)^{\frac{1}{p}} \mathrm{e}_{\lambda_{n}}\right)_{n}$ is equivalent to the canonical basis of $\ell^{p}$ in $L^{p}(0,1)$.

- The sequence $\left(\mathrm{e}_{\lambda_{n}}\right)_{n}$ is equivalent to the summing basis of $c$ in $C([0,1])$.

- $\Lambda$ is lacunary.

In other words, when $\Lambda$ is lacunary, there exist $C_{1}, C_{2}>0$ such that

$$
C_{1}\left(\sum_{k \geq 0}\left|a_{k}\right|^{p}\right)^{\frac{1}{p}} \leq\left\|\sum_{k \geq 0} a_{k}\left(p \lambda_{k}+1\right)^{\frac{1}{p}} \mathrm{e}_{\lambda_{k}}\right\|_{L^{p}} \leq C_{2}\left(\sum_{k \geq 0}\left|a_{k}\right|^{p}\right)^{\frac{1}{p}}
$$

and

$$
C_{1} \sup _{m \geq 0}\left|\sum_{k=0}^{m} a_{k}\right| \leq\left\|\sum_{k \geq 0} a_{k} \mathrm{e}_{\lambda_{k}}\right\|_{\infty} \leq \sup _{m \geq 0}\left|\sum_{k=0}^{m} a_{k}\right|
$$


3.1. Müntz spaces and Bergman spaces. The Gurariy-Macaev theorem states that, when $\Lambda$ is lacunary, $M_{\Lambda}^{p}$ is isomorphic to $\ell^{p}$ and looks like the (whole) Bergman space $\mathcal{A}^{p}$ from the geometry of Banach space viewpoint, but not only: the norms on $L^{p}(0,1)$ and on $\mathcal{A}^{p}$ are equivalent for lacunary Taylor series, but not for every Taylor series.

Proposition 3.2. Let $p \geq 1$.

(i) There exists $f \in \mathcal{A}^{p}$ such that $f_{\mid(0,1)} \notin L^{p}(0,1)$.

(ii) Let $\Lambda \subset \mathbb{N}$ be a lacunary set. There exist $C, C^{\prime}>0$ such that for every polynomial $f$ spanned by the functions $z \rightarrow z^{\lambda}$ with $\lambda \in \Lambda$, we have

$$
C\|f\|_{\mathcal{A}^{p}} \leq\|f\|_{L^{p}(0,1)} \leq C^{\prime}\|f\|_{\mathcal{A}^{p}}
$$

Proof. ( $i$ ) follows quickly from the fact that the contrary would mean that the Carleson embedding $\mathcal{A}^{p} \hookrightarrow L^{p}(\nu)$ would be bounded, where the measure $\nu$ is defined by $\nu(B)=m(B \cap(0,1)$ ) (where $B$ is a Borel subset of $\overline{\mathbb{D}}$ ). Hence this measure $\nu$ would be 2-Carleson, but clearly, considering the Carleson window $S(1, h)=\{z \in$ $\mathbb{D}|| 1-z \mid \leq h\}$, we have $\nu(S(1, h))=h$, which is not $O\left(h^{2}\right)$.

(ii) follows from Gurariy-Macaev theorem. Indeed for every $\theta \in(0,1)$, we have for any polynomial $f=\sum_{k} a_{k} z^{\lambda_{k}}$

$$
C_{1}\left(\sum_{k \geq 0} \frac{\left|a_{k}\right|^{p}}{p \lambda_{k}+2}\right)^{\frac{1}{p}} \leq\left\|\sum_{k \geq 0} a_{k} \mathrm{e}^{2 i \pi \lambda_{k} \theta} \mathrm{e}_{\lambda_{k}+\frac{1}{p}}\right\|_{L^{p}(0,1)} \leq C_{2}\left(\sum_{k \geq 0} \frac{\left|a_{k}\right|^{p}}{p \lambda_{k}+2}\right)^{\frac{1}{p}}
$$

where $C_{1}$ and $C_{2}$ are the constants given by Th.3.1.

Now, recall that $\|f\|_{\mathcal{A}^{p}}^{p}=2 \int_{0}^{1} \int_{0}^{1}\left|\sum_{k \geq 0} a_{k} r^{\lambda_{k}} \mathrm{e}^{2 i \pi \lambda_{k} \theta}\right|^{p} r d r d \theta$ and we get

$$
\left\|\sum_{k} a_{k} z^{\lambda_{k}}\right\|_{\mathcal{A}^{p}} \approx\left(\sum_{k} \frac{\left|a_{k}\right|^{p}}{p \lambda_{k}+2}\right)^{\frac{1}{p}} \approx\left(\sum_{k} \frac{\left|a_{k}\right|^{p}}{p \lambda_{k}+1}\right)^{\frac{1}{p}} \approx\|f\|_{L^{p}(0,1)}
$$

applying again Th.3.1.

It is not known whether $M_{\Lambda}^{p}$ is also isomorphic to $\ell^{p}$ for any $\Lambda$. It is the case if $\Lambda$ is a finite union of lacunary sequence (i.e. quasi-lacunary, see [10]), but in that case, the isomorphism is not the "natural" one underlying in Gurariy-Macaev theorem as soon as $\Lambda$ is not lacunary.

3.2. Müntz spaces and Hardy spaces. Since $\nu(B)=m(B \cap(0,1))$ (where $B$ is a Borel subset of $\overline{\mathbb{D}}$ ) defines a Carleson measure on $\overline{\mathbb{D}}$, we have the following inequality for every Taylor polynomial $f$ :

$$
\|f\|_{L^{p}(0,1)} \lesssim\|f\|_{H^{p}} .
$$

It is immediate that the norms are not equivalent: the $L^{p}$ norm of $z^{n}$ satisfies $\left\|z^{n}\right\|_{p} \approx 1 / n^{\frac{1}{p}}$ whereas $\left\|z^{n}\right\|_{H^{p}}=1$.

3.3. Back to Gurariy-Macaev theorem. Let us mention that an almost isometric version of Gurariy-Macaev theorem is proved in [7] (see [8] for cases $p \in\{1,+\infty\}$ too, and [6] for an alternative approach in the hilbertian framework for complex exponents), which is relative to the arithmetical condition of super-lacunarity: an increasing sequence of positive numbers $\left(p_{n}\right)_{n}$ is super-lacunary if $\lim _{n \rightarrow+\infty} \frac{p_{n+1}}{p_{n}}=+\infty$. Before giving the statement of the theorem, we recall

Definition 3.3. Let $\left(x_{n}\right)_{n \geq 1}$ be a normalized sequence in a Banach space $X$. 
- The sequence $\left(x_{n}\right)_{n \geq 1}$ is almost isometric to the canonical basis of $\ell^{p}$ in $X$ if

$$
\left(1-\varepsilon_{n}\right)\left(\sum_{k \geq n}\left|a_{k}\right|^{p}\right)^{\frac{1}{p}} \leq\left\|\sum_{k \geq n} a_{k} x_{k}\right\|_{X} \leq\left(1+\varepsilon_{n}\right)\left(\sum_{k \geq n}\left|a_{k}\right|^{p}\right)^{\frac{1}{p}}
$$

where $\varepsilon_{n} \rightarrow 0$

- The sequence $\left(x_{n}\right)_{n \geq 1}$ is almost isometric to the summing basis of $c$ in $X$ if

$$
\left(1-\varepsilon_{n}\right) \sup _{m \geq n}\left|\sum_{k=n}^{m} a_{k}\right| \leq\left\|\sum_{k \geq n} a_{k} x_{k}\right\|_{X} \leq\left(1+\varepsilon_{n}\right) \sup _{m \geq n}\left|\sum_{k=n}^{m} a_{k}\right|
$$

where $\varepsilon_{n} \rightarrow 0$

When $X$ is a Hilbert space (and $p=2$ ), we usually rather say that the sequence $\left(x_{n}\right)_{n \geq 1}$ is asymptotically orthonormal.

We mention

Theorem 3.4. $[7,8]$ Let $p \geq 1$. TFAE

- The normalized sequence $\left(\left(p \lambda_{n}+1\right)^{\frac{1}{p}} \mathrm{e}_{\lambda_{n}}\right)_{n}$ is almost isometric to the canonical basis of $\ell^{p}$ in $L^{p}(0,1)$

- The sequence $\left(\mathrm{e}_{\lambda_{n}}\right)_{n}$ is almost isometric to the summing basis of c in $C([0,1])$.

- $\Lambda$ is super-lacunary.

See [7] and [8] for a proof, but we incorporate below a very short and elementary proof in the case $p=2$ (less technical than the original one) and complete it with the equivalence to a (strong) variant of almost isometry. This is the counterpart in Müntz spaces of the same argument given in [6] for a particular case of a result of Volberg on model spaces.

Proposition 3.5. TFAE

(1) There exists $n_{0} \geq 1$ and a sequence $\left(\varepsilon_{n}\right)_{n \geq n_{0}}$ in $(0,1)$ with $\varepsilon_{n} \rightarrow 0$ such that

$$
\left(\sum_{n \geq n_{0}}\left(1-\varepsilon_{n}\right)\left|a_{n}\right|^{2}\right)^{\frac{1}{2}} \leq\left\|\sum_{n \geq n_{0}} a_{n}\left(2 \lambda_{n}+1\right)^{\frac{1}{2}} \mathrm{e}_{\lambda_{n}}\right\|_{L^{2}} \leq\left(\sum_{n \geq n_{0}}\left(1+\varepsilon_{n}\right)\left|a_{n}\right|^{2}\right)^{\frac{1}{2}}
$$

(2) There exists a sequence $\varepsilon_{n} \rightarrow 0$ such that

$$
\left(1-\varepsilon_{n}\right)\left(\sum_{k \geq n}\left|a_{k}\right|^{2}\right)^{\frac{1}{2}} \leq\left\|\sum_{k \geq n} a_{k}\left(2 \lambda_{k}+1\right)^{\frac{1}{2}} \mathrm{e}_{\lambda_{k}}\right\|_{L^{2}} \leq\left(1+\varepsilon_{n}\right)\left(\sum_{k \geq n}\left|a_{k}\right|^{2}\right)^{\frac{1}{2}}
$$

(3) $\Lambda$ is super-lacunary.

Proof. $(1) \Rightarrow(2)$ is clear.

$(2) \Rightarrow(3)$ it is not new but we give the following easy argument for completeness: for every $t>0$, we have

$$
\begin{aligned}
\left(1+\varepsilon_{n}\right)\left(1+t^{2}\right)^{\frac{1}{2}} & \geq\left\|\left(2 \lambda_{n}+1\right)^{\frac{1}{2}} \mathrm{e}_{\lambda_{n}}+t\left(2 \lambda_{n+1}+1\right)^{\frac{1}{2}} \mathrm{e}_{\lambda_{n+1}}\right\| \\
& \geq\left\langle\left(2 \lambda_{n}+1\right)^{\frac{1}{2}} \mathrm{e}_{\lambda_{n}}+t\left(2 \lambda_{n+1}+1\right)^{\frac{1}{2}} \mathrm{e}_{\lambda_{n+1}},\left(2 \lambda_{n}+1\right)^{\frac{1}{2}} \mathrm{e}_{\lambda_{n}}\right\rangle \\
& \geq 1+2 t \frac{\sqrt{\frac{\lambda_{n+1}}{\lambda_{n}}}}{1+\frac{\lambda_{n+1}}{\lambda_{n}}}
\end{aligned}
$$

which implies that $\frac{\lambda_{n+1}}{\lambda_{n}} \rightarrow+\infty$. 
$(3) \Rightarrow(1)$ Here is the novelty. Let us fix a super-lacunary sequence $\left(\lambda_{n}\right)$, which means that $\frac{\lambda_{n+1}}{\lambda_{n}} \rightarrow+\infty$. Equivalently $q_{n}=\left(2 \lambda_{n}+1\right)^{\frac{1}{2}}$ is super-lacunary. Now we introduce $\rho_{n}=\inf _{j \geq n} \frac{q_{j+1}}{q_{j}}$ and $\delta_{n}=\rho_{n-1}^{-\frac{1}{3}}>0$. Choose $n_{0}$ such that $\delta_{n_{0}}<1 / 2$.

Now take any finitely supported sequence of scalars $\left(a_{k}\right)$ and develop

$$
\begin{aligned}
\left\|\sum_{k \geq n_{0}} a_{k}\left(2 \lambda_{k}+1\right)^{\frac{1}{2}} \mathrm{e}_{\lambda_{k}}\right\|^{2} & =\sum_{k, l \geq n_{0}} a_{k} \overline{a_{l}} q_{k} q_{l}\left\langle\mathrm{e}_{\lambda_{k}}, \mathrm{e}_{\lambda_{l}}\right\rangle \\
& =\sum_{k, l \geq n_{0}} 2 a_{k} \overline{a_{l}} \frac{q_{k} q_{l}}{q_{k}^{2}+q_{l}^{2}} \\
& =\|a\|_{2}^{2}+\sum_{\substack{k, l \geq n_{0} \\
k \neq l}} 2 a_{k} \overline{a_{l}} \frac{q_{k} q_{l}}{q_{k}^{2}+q_{l}^{2}}
\end{aligned}
$$

We focus on the sum in the right hand side of $(*)$. Write $a_{n}^{\prime}=a_{n} \delta_{n}$, so that

$$
\sum_{\substack{k, l \geq n_{0} \\ k \neq l}} 2 a_{k} \overline{a_{l}} \frac{q_{k} q_{l}}{q_{k}^{2}+q_{l}^{2}}=\sum_{\substack{k, l \geq n_{0} \\ k \neq l}} 2 a_{k}^{\prime} \overline{a_{l}^{\prime}} \frac{q_{k} q_{l}}{q_{k}^{2}+q_{l}^{2}} \delta_{k}^{-1} \delta_{l}^{-1}
$$

Using the inequality $\left|2 a_{k}^{\prime} \overline{a_{l}^{\prime}}\right| \leq\left|a_{k}^{\prime}\right|^{2}+\left|a_{l}^{\prime}\right|^{2}$, we get

$$
\left|\sum_{\substack{k, l \geq n_{0} \\ k \neq l}} 2 a_{k} \overline{a_{l}} \frac{q_{k} q_{l}}{q_{k}^{2}+q_{l}^{2}}\right| \leq 2\left\|a^{\prime}\right\|_{2}^{2} \sup _{l \geq n_{0}} \sum_{\substack{k \geq n_{0} \\ k \neq l}} \frac{q_{k} q_{l}}{q_{k}^{2}+q_{l}^{2}} \delta_{k}^{-1} \delta_{l}^{-1} .
$$

For every $l \geq n_{0}$, we have $\sum_{\substack{k \geq n_{0} \\ k \neq l}} \frac{q_{k} q_{l}}{q_{k}^{2}+q_{l}^{2}} \delta_{k}^{-1} \delta_{l}^{-1} \leq 2 \sum_{k>l} \frac{q_{l}}{q_{k}} \delta_{k}^{-1} \delta_{l}^{-1}$, but

$$
\forall k>l, \quad q_{k} \geq \rho_{k-1} \ldots \rho_{l} q_{l} \quad \text { and } \quad \frac{q_{l}}{q_{k}} \delta_{k}^{-1} \delta_{l}^{-1} \leq \frac{\rho_{k-1}^{\frac{1}{3}} \rho_{l-1}^{\frac{1}{3}}}{\rho_{k-1} \ldots \rho_{l}} \leq \frac{1}{\rho_{l}^{\frac{1}{3}(k-l)}} .
$$

Hence

$$
\left|\sum_{\substack{k, l \geq n_{0} \\ k \neq l}} 2 a_{k} \overline{a_{l}} \frac{q_{k} q_{l}}{q_{k}^{2}+q_{l}^{2}}\right| \leq \frac{4}{\rho_{n_{0}}^{\frac{1}{3}}-1}\left\|a^{\prime}\right\|_{2}^{2} .
$$

Writing $\varepsilon_{n}=\frac{4}{\rho_{n_{0}}^{\frac{1}{3}}-1} \delta_{n}^{2}$, we easily check that $\varepsilon_{n} \in(0,1)$ and we get the result.

Let us point out that if one want to prove directly $(3) \Rightarrow(2)$, the preceding argument is easily simplified (avoiding the trick with $a_{k}^{\prime}$ and $\delta_{k}$ ). Actually in that case, we can get directly an upper bound estimate in $(* *)$ with $4\|a\|_{2}^{2} /\left(\rho_{n_{0}}-1\right)$.

\section{Bloch type spaces AND inequalities}

In this section, we introduce the Müntz-Bloch space $\mathcal{B}_{\Lambda}^{\alpha}$, and study the link with classical Müntz spaces.

Definition 4.1. Let $\alpha>0$. We introduce the space $\mathcal{B}^{\alpha}$ as the space

$$
\mathcal{B}^{\alpha}=\left\{f \in C^{1}([0,1))\left|\sup _{[0,1)}(1-t)^{\alpha}\right| f^{\prime}(t) \mid<\infty\right\}
$$


equipped with the norm

$$
\|f\|_{\mathcal{B}^{\alpha}}=|f(0)|+\sup _{[0,1)}(1-t)^{\alpha}\left|f^{\prime}(t)\right|
$$

It is easy to check that $\mathcal{B}^{\alpha}$ is a Banach space. When $\alpha=1$, we shall simply write $\mathcal{B}$ instead of $\mathcal{B}^{1}$.

Let us focus on the behavior of monomials in this space. For every $\lambda \geq 1$, the function $\mathrm{e}_{\lambda}$ belongs to $\mathcal{B}^{\alpha}$ with

$$
\left\|\mathrm{e}_{\lambda}\right\|_{\mathcal{B}^{\alpha}}=\frac{\lambda \alpha^{\alpha}(\lambda-1)^{\lambda-1}}{(\lambda+\alpha-1)^{\lambda+\alpha-1}}
$$

and in particular $\left\|\mathrm{e}_{\lambda}\right\|_{\mathcal{B}}=\left(1-\frac{1}{\lambda}\right)^{\lambda-1}$.

We also mention that

$$
\lim _{\lambda \rightarrow+\infty}\left\|\mathrm{e}_{\lambda}\right\|_{\mathcal{B}^{\alpha}}^{\frac{1}{\lambda}}=1
$$

From our version of the Clarkson-Erdös-Schwartz theorem (Th.2.4), we get a nice Taylor type expansion for functions belonging to $\mathcal{B}_{\Lambda}^{\alpha}={\overline{M_{\Lambda}}}^{\mathcal{B}^{\alpha}}$.

Theorem 4.2. Let $\alpha>0$ and $\Lambda=\left\{\lambda_{n}\right\}$ satisfying the gap condition (GC) and $\sum 1 / \lambda_{n}<+\infty$.

For every $f \in \mathcal{B}_{\Lambda}^{\alpha}$, we have

$$
\exists\left(a_{n}\right)_{n} \in \mathbb{C}^{\mathbb{N}}, \quad f(t)=\sum_{n} a_{n} t^{\lambda_{n}} \quad \text { for every } t \in[0,1) .
$$

Proof. When $\alpha \in(0,2)$, it suffices to apply Th.2.4. We only have to justify that there exists some $C>0$ such that $\|f\|_{1} \leq C\|f\|_{\mathcal{B}^{\alpha}}$. Indeed, fix $f \in \mathcal{B}^{\alpha}$.

For every $x \in[0,1)$, we can write: $f(x)=f(0)+\int_{0}^{x} f^{\prime}(s) d s$ so that

$$
\|f\|_{1} \leq|f(0)|+\int_{0}^{1}\left(\int_{0}^{x}\left|f^{\prime}(s)\right| d s\right) d x=|f(0)|+\int_{0}^{1}(1-s)\left|f^{\prime}(s)\right| d s
$$

via Fubini.

Now we have

$\int_{0}^{1}(1-s)\left|f^{\prime}(s)\right| d s \leq \sup _{(0,1)}(1-t)^{\alpha}\left|f^{\prime}(t)\right| \int_{0}^{1}(1-s)^{1-\alpha} d s=\frac{1}{2-\alpha} \sup _{(0,1)}(1-t)^{\alpha}\left|f^{\prime}(t)\right|$

which gives the conclusion when $\alpha<2$.

Of course, $t \in[0,1) \mapsto \frac{1}{1-t}$ belongs to $\mathcal{B}^{2}$, but not to $L^{1}(0,1)$. To prove the theorem when $\alpha \geq 2$, we use another argument (which would work for any value of $\alpha$ ). Fix $\varphi$ in the unit ball of the dual of $\mathcal{B}^{\alpha}$. Fix a polynomial $P=\sum_{n} a_{n} \mathrm{e}_{\lambda_{n}}$. The map

is continuous.

$$
\rho \in[0,1] \longmapsto \sum_{n} a_{n} \rho^{\lambda_{n}} \varphi\left(\mathrm{e}_{\lambda_{n}}\right)
$$

But we already proved the Clarkson-Erdös-Schwartz theorem for $M_{\Lambda}^{\infty}$ and we get for every $\varepsilon>0$ the existence of $C_{\varepsilon}$ (depending on $\Lambda$ and $\varepsilon$ only) such that

$$
\left|a_{n} \varphi\left(\mathrm{e}_{\lambda_{n}}\right)\right| \leq C_{\varepsilon}(1+\varepsilon)^{\lambda_{n}} \sup _{\rho \in[0,1]}\left|\sum_{n} a_{n} \rho^{\lambda_{n}} \varphi\left(\mathrm{e}_{\lambda_{n}}\right)\right|
$$


and since $\left|\sum_{n} a_{n} \rho^{\lambda_{n}} \varphi\left(\mathrm{e}_{\lambda_{n}}\right)\right| \leq\left\|\sum_{n} a_{n} \rho^{\lambda_{n}} \mathrm{e}_{\lambda_{n}}\right\|_{\mathcal{B}^{\alpha}}$, we obtain by Hahn-Banach

$$
\left|a_{n}\right|\left\|\mathrm{e}_{\lambda_{n}}\right\|_{\mathcal{B}^{\alpha}} \leq C_{\varepsilon}(1+\varepsilon)^{\lambda_{n}} \sup _{\rho \in(0,1)}\|P(\rho .)\|_{\mathcal{B}^{\alpha}}
$$

But

$$
\|P(\rho .)\|_{\mathcal{B}^{\alpha}}=|P(0)|+\sup _{(0,1)}(1-t)^{\alpha} \rho\left|P^{\prime}(\rho t)\right| \leq|P(0)|+\sup _{(0,1)}(1-\rho t)^{\alpha}\left|P^{\prime}(\rho t)\right| \leq\|P\|_{\mathcal{B}^{\alpha}} .
$$

We get $\left|a_{n}\right|\left\|\mathrm{e}_{\lambda_{n}}\right\|_{\mathcal{B}^{\alpha}} \leq C_{\varepsilon}(1+\varepsilon)^{\lambda_{n}}\|P\|_{\mathcal{B}^{\alpha}}$ and thanks to (7), we even have some $C_{\varepsilon}^{\prime}$ (depending on $\Lambda$ and $\varepsilon$ only) such that

$$
\left|a_{n}\right| \leq C_{\varepsilon}^{\prime}(1+\varepsilon)^{\lambda_{n}}\|P\|_{\mathcal{B}^{\alpha}}
$$

Now, we only have to end the proof like for Th.2.4.

Let us point out that $\left\|\lambda^{\alpha-1} \mathrm{e}_{\lambda}\right\|_{\mathcal{B}^{\alpha}} \sim \alpha^{\alpha} \mathrm{e}^{-\alpha}$ when $\lambda \rightarrow+\infty$. In particular, we have the asymptotic behavior $\left\|(p \lambda+1)^{1 / p} \mathrm{e}_{\lambda}\right\|_{\mathcal{B}^{1+\frac{1}{p}}} \sim \frac{(p+1)^{1+\frac{1}{p}}}{p \mathrm{e}^{1+\frac{1}{p}}} \cdot$ Therefore, for any $p \geq 1$, we cannot have the (continuous) embedding $M_{\Lambda}^{p} \subset \mathcal{B}^{\alpha}$ when $\alpha<1+1 / p$. The preceding phenomenom occurs too in the case $p=+\infty$ and $M_{\Lambda}^{\infty} \subset \mathcal{B}^{1}$ is the best we could expect. It is then natural to ask the following questions:

Problem 1. Let $p \in[1,+\infty]$ and $\Lambda$ satisfying both Müntz condition and (GC). Do we have $M_{\Lambda}^{p} \subset \mathcal{B}^{1+\frac{1}{p}}$ (continuously) ?

In other words, does there exist $C>0$, such that, for every $f \in M_{\Lambda}^{p}$,

$$
\forall t \in(0,1), \quad\left|f^{\prime}(t)\right| \leq \frac{C}{(1-t)^{1+\frac{1}{p}}}\|f\|_{p} \quad \text { ? }
$$

Another natural question is closely related in the infinite case:

Problem 2. Let $\Lambda$ satisfying the Müntz condition and (GC).

Does the derivatives of the functions of the Müntz space $M_{\Lambda}^{\infty}$ belong to the weak- $L^{1}$ space $L_{w}^{1}(0,1)$ ?

We give an answer to Problem 1: Theorem 4.3 (see also the remark after its statement) shows that there exists $\Lambda$ such that, for any value of $p$, the answer to the question in problem 1 is negative. We give also an answer to Problem 2 in Theorem 4.5. Actually we show that for both questions, there exists an arithmetical constraint on $\Lambda$. A negative answer to Problem 2 implies a negative to Problem 1 for $p=+\infty$. Nevertheless, we shall give separate proofs, through it relies on the same kind of ideas, the negative answer to Problem 1 being a bit faster to prove (and stated for every $p \in[1,+\infty])$.

We wish to mention that actually a negative answer to problem 1 in the case $p=+\infty$ can also be found in the monograph [4]: see E.9 p.318. There, another arithmetical restriction is given in this case: $\lambda_{n} \geq c \rho^{n}$ for some $c>0$ and some $\rho>1$. Our argument will be independent, more elementary (according to us), fits to any value of $p$, but leads to a different kind of arithmetical condition.

Let us add that we have a positive answer to problem 1 when $\Lambda$ is lacunary: it is easy to see (and more or less part of the folklore). It follows from the fact that in this case: $f(t)=\sum_{n} a_{n} t^{\lambda_{n}}$ where $t \in(0,1)$ and $\left|a_{n}\right| \leq C \lambda_{n}^{\frac{1}{p}}\|f\|_{p}$ for some constant 
$C$ depending on the lacunarity of $\Lambda$ only (it is a direct consequence of Th.3.1 for instance). Then it suffices to see that for every $t \in[0,1)$ and $\alpha>0$, we have

$$
\sum \lambda_{n}^{\alpha} t^{\lambda_{n}-1} \lesssim \frac{1}{(1-t)^{\alpha}}
$$

This can be viewed as the counterpart in our framework of the following well known fact: every function of $H^{\infty}$ belongs to the (classical) Bloch space of functions $f$, analytic on $\mathbb{D}$, such that $\sup _{z \in \mathbb{D}}(1-|z|)\left|f^{\prime}(z)\right|$.

On the other hand, we must mention too that it was stated recently in [13], [14], and [15] that problem 1 and 2 always have a positive answer. Actually the mistake in these papers is the following: a Taylor series with radius 1 whose restriction to $[0,1)$ has a limit at point 1 needs not to be bounded on the disk centered at $1 / 2$, with radius $1 / 2$. These inequalities were the conerstone of the proof of the existence of a Schauder basis for every Müntz spaces. Therefore the counterexamples below show that the problem of existence of basis for Müntz spaces is still completely open.

We give now our counterexamples.

Theorem 4.3. Let $p \in[1,+\infty]$ and $\Lambda$ satisfying the Müntz condition such that there exists an increasing sequence $\left(a_{n}\right)_{n}$ with $a_{n} \rightarrow+\infty$, such that $\bigcup_{n \geq 1}\left\{a_{n}, \ldots, n a_{n}\right\} \subset \Lambda$.

Then

- for every $C>0$, there exists $f \in M_{\Lambda}^{p}$ satisfying

$$
\sup _{t \in(0,1)}(1-t)^{1+\frac{1}{p}}\left|f^{\prime}(t)\right|>C\|f\|_{p} .
$$

- $M_{\Lambda}^{p} \not \subset \mathcal{B}_{1+\frac{1}{p}}$

This theorem shows that there exists a set $\Lambda$ of integers such that, for every $p \in[1,+\infty]$, the answer to problem 1 is negative: choose a sequence of integers such that $a_{n+1}>n a_{n}$ then the Müntz and the gap conditions are fulfilled and the set $\Lambda=\bigcup_{n \geq 1}\left\{a_{n}, \ldots, n a_{n}\right\}$ makes the job.

Proof. We begin with the following simple fact

Fact: given $A>0$, there exists a polynomial $Q$ such that $|Q(t)| \leq t$ and $\left|Q^{\prime}(1 / \mathrm{e})\right| \geq A$.

One can argue for example by standard abstract functional analysis: the derivation at point $1 /$ e cannot be bounded on $\left(\mathbb{R}[X],\|\cdot\|_{\infty}\right)$. This provides some polynomial $P$ such that $\|P\|_{\infty} \leq 1$ and $\left|P^{\prime}(1 / \mathrm{e})\right| \geq A$. Actually such a polynomial would suffice to work with when $p=+\infty$ but we shall need the condition $|Q(t)| \leq t$ on $[0,1]$ in the case $p \in[1,+\infty)$. It suffices to take a polynomial $P$ associated to $\mathrm{e}(A+1)$ (instead of $A$ ), then take $Q(X)=X . P(X)$. The fact is proved.

Now, assume that $p \in[1,+\infty]$ is fixed and there exists $C>0$ such that

$$
\forall t \in(0,1), \quad\left|f^{\prime}(t)\right| \leq \frac{C}{(1-t)^{1+\frac{1}{p}}}\|f\|_{p} .
$$

Take $A=2 C$ e and apply the fact. Denote by $d$ the degree of our polynomial $Q$, consider the function $f(t)=Q\left(t^{a_{n}}\right)$ for $t \in[0,1]$. This function $f$ belongs to $M_{\Lambda}$ when $n \geq d$. We test the inequality at point $t=1-\frac{1}{a_{n}}$ for $n$ large enough and we 
get

$$
a_{n}\left(1-\frac{1}{a_{n}}\right)^{a_{n}-1}\left|Q^{\prime}\left(\left(1-\frac{1}{a_{n}}\right)^{a_{n}}\right)\right| \leq C a_{n}^{1+\frac{1}{p}}\|f\|_{p}
$$

But, for $p=+\infty$, we already know that $\|f\|_{\infty} \leq 1$ and, when $p$ is finite, we have $\|f\|_{p}^{p} \leq \int_{0}^{1} t^{p a_{n}} d t=\frac{1}{p a_{n}+1} \leq \frac{1}{a_{n}}$ so in every case,

$$
\left(1-\frac{1}{a_{n}}\right)^{a_{n}-1}\left|Q^{\prime}\left(\left(1-\frac{1}{a_{n}}\right)^{a_{n}}\right)\right| \leq C
$$

Letting $n$ going to infinity, we get $\mathrm{e}^{-1}\left|Q^{\prime}(1 / \mathrm{e})\right| \leq C$ and a contradiction.

Now we focus on Problem 2. When $\Lambda$ is lacunary, we already mentioned that every $f \in M_{\Lambda}^{\infty}$ belongs to $\mathcal{B}^{1}$, so that its derivative $f^{\prime}$ belongs to $L_{w}^{1}(0,1)$. For our counterexample, we first need the following lemma.

Lemma 4.4. Let $\varepsilon \in(0,1)$ and $A>0$. There exists a polynomial $P$ such that $P(0)=0,\|P\|_{\infty}=1$ and the set $E=\left\{x \in(0,1)|| P^{\prime}(x) \mid<A\right\}$ has Lebesgue measure less than $\varepsilon$.

Proof. First consider a function $h \in C^{1}([0,1])$ such that $0 \leq h \leq 1$ with $h(0)=0$, $\|h\|_{\infty}=1$ and $\left|h^{\prime}\right|=2 A+1$ everywhere except on a finite number (say $N \approx A$ ) of intervals (centered at points $k /(2 A+1)$ ) whose length is $\varepsilon / N$. Denote $\mathcal{N}_{\varepsilon}$ the union of these $N$ intervals. $\mathcal{N}_{\varepsilon}$ has Lebesgue measure less than $\varepsilon$.

Now there exists some polynomial $Q_{1}$ such that $\left\|Q_{1}-h^{\prime}\right\|_{\infty} \leq 1$. Take the polynomial $Q$ such that $Q^{\prime}=Q_{1}$ and $Q(0)=0$. We have $\|Q-h\|_{\infty} \leq 1$ so that $\|Q\|_{\infty} \leq 2$. Moreover, for every $x \notin \mathcal{N}_{\varepsilon},\left|Q^{\prime}(x)\right| \geq\left|h^{\prime}(x)\right|-1 \geq 2 A$.

At last $P=Q /\|Q\|_{\infty}$ is the desired polynomial.

Theorem 4.5. Assume that there exists an increasing sequence $\left(a_{n}\right)_{n}$ with $a_{n} \rightarrow$ $+\infty$, such that $\bigcup_{n \geq 1}\left\{a_{n}, \ldots, n a_{n}\right\} \subset \Lambda$.

Then there exists $f \in M_{\Lambda}^{\infty}$ such that $f^{\prime} \notin L_{w}^{1}(0,1)$.

Using the same set than in the remark after Theorem 4.3, we have a negative answer to Problem 2.

Proof. The proof follows the spirit of the one of Th.4.3.

Claim: for any $C>0$, there exists some $f \in M_{\Lambda}^{\infty}$ with uniform norm equal to 1 such that $\left\|f^{\prime}\right\|_{L_{w}^{1}(0,1)} \geq C$.

We fix $C>0$ and apply Lemma 4.4 with $A=4 \mathrm{e} C$ and $\varepsilon=1 / 2 \mathrm{e}$. We are given a norm 1 polynomial $P$ with some degree $d_{0} \geq 1$ such that

$$
E=\left\{x \in(0,1)|| P^{\prime}(x) \mid<A\right\}
$$

has Lebesgue measure less than $\varepsilon$.

We consider now $d \geq d_{0}$ and $f(t)=P\left(t^{a_{d}}\right)$ for $t \in[0,1]$. It belongs to $M_{\Lambda}^{\infty}$, satisfies $f(0)=0$ and has norm 1 . We may assume that $a_{d} \geq 1$.

We have

$$
\left\{t \in(0,1) ;\left|f^{\prime}(t)\right| \geq 4 a_{d} C\right\}=\left\{t \in(0,1) ; t^{a_{d}-1}\left|P^{\prime}\left(t^{a_{d}}\right)\right| \geq 4 C\right\}
$$


hence

$$
\begin{aligned}
m\left(\left\{t \in(0,1) ;\left|f^{\prime}(t)\right| \geq 4 a_{d} C\right\}\right) \geq & m\left(\left\{t \in\left(\exp \left(-1 / a_{d}\right), 1\right) ;\left|P^{\prime}\left(t^{a_{d}}\right)\right| \geq 4 \mathrm{e} C\right\}\right) \\
= & 1-\exp \left(-\frac{1}{a_{d}}\right) \\
& -m\left(\left\{t \in\left(\exp \left(-1 / a_{d}\right), 1\right) ; t^{a_{d}} \in E\right\}\right)
\end{aligned}
$$

But, using the change of variable formula,

$$
m\left(\left\{t \in\left(\exp \left(-1 / a_{d}\right), 1\right) ; t^{a_{d}} \in E\right\}\right)=\frac{1}{a_{d}} \int_{1 / \mathrm{e}}^{1} \mathbb{1}_{E}(u) u^{\frac{1}{a_{d}}-1} d u \leq \frac{\mathrm{e}}{a_{d}} m(E) \leq \frac{1}{2 a_{d}} .
$$

We get (when $d \rightarrow+\infty$ )

$$
4 a_{d} C m\left(\left\{t \in(0,1) ;\left|f^{\prime}(t)\right| \geq 4 a_{d} C\right\}\right) \geq 4 a_{d} C\left(1-\exp \left(-1 / a_{d}\right)-\frac{1}{2 a_{d}}\right) \sim 2 C
$$

so that, for $d$ large enough,

$$
\left\|f^{\prime}\right\|_{L_{w}^{1}(0,1)} \geq 4 a_{d} C m\left(\left\{t \in(0,1) ;\left|f^{\prime}(t)\right| \geq 4 a_{d} C\right\}\right) \geq C
$$

which was our claim.

We can conclude the proof by a standard argument via the closed graph theorem. Indeed, if the conclusion of our theorem were not true, the map

$$
\begin{array}{cl}
M_{\Lambda}^{\infty} & \stackrel{J}{\longrightarrow} L_{w}^{1}(0,1) \\
f & \longmapsto
\end{array}
$$

would be defined.

Let us show that $J$ has a closed graph in that situation. Let $\left(f_{n}\right)$ be a sequence in $M_{\Lambda}^{\infty}$ such that $f_{n} \rightarrow f$ in $M_{\Lambda}^{\infty}$ and $f_{n}^{\prime} \rightarrow g$ in $L_{w}^{1}(0,1)$. Then we know that $f_{n}^{\prime} \rightarrow f^{\prime}$ uniformly on compact subsets of $[0,1)$ hence for every $\varepsilon>0$ and every $y \in[0,1)$

$$
\begin{aligned}
& m\left(\left\{t \in[0, y]|| f^{\prime}(t)-g(t) \mid \geq \varepsilon\right\}\right) \leq m\left(\left\{t \in[0, y]|| f^{\prime}(t)-f_{n}^{\prime}(t) \mid \geq \varepsilon / 2\right\}\right)+ \\
& m\left(\left\{t \in[0, y]|| f_{n}^{\prime}(t)-g(t) \mid \geq \varepsilon / 2\right\}\right) \\
& \leq \frac{2}{\varepsilon}\left\|f_{n}^{\prime}-f^{\prime}\right\|_{L^{1}(0, y)}+\frac{2}{\varepsilon}\left\|f_{n}^{\prime}-g\right\|_{L_{w}^{1}}
\end{aligned}
$$

Letting $n$ going to infinity, we get that the measure of $\left\{t \in[0, y]|| f^{\prime}(t)-g(t) \mid \geq \varepsilon\right\}$ is equal to 0 for every $\varepsilon>0$. Therefore $f^{\prime}=g$ a.e. on $[0, y]$ hence on $[0,1)$ since $y$ was arbitrary. The graph of $J$ is closed as announced.

The closed graph theorem $\left(L_{w}^{1}(0,1)\right.$ is a quasi-Banach space) implies that the map $J$ is continuous, which contradicts our claim.

Acknowledgment. We wish to thank the referee for her/his suggestions in order to improve the quality of this paper.

\section{REFERENCES}

[1] I. AlAlam, G. Habib, P. Lefèvre, F. Maalouf, Essential norms of Volterra and Cesàro operators on Müntz spaces, Colloq. Math. DOI: 10.4064/cm7100-1-2017.

[2] J.M.Almira, Müntz type theorems I, Surveys in Approximation Theory, vol. 3, 2007, 152-194.

[3] P. Borwein, T. Erdélyi, The full Müntz theorem in $C[0,1]$ and $L^{1}[0,1]$, J. London Math. Soc. (2) 54 (1996), no. 1, 102-110.

[4] P. Borwein, T. Erdélyi, Polynomials and Polynomial Inequalities, Graduate Texts in Mathematics, 161. Springer-Verlag, New York, 1995. 
[5] I. Chalendar, E. Fricain, D. Timotin, Embeddings theorems for Müntz spaces, Ann. Inst. Fourier, 61 (2011), 2291-2311.

[6] E. Fricain, P. Lefèvre, Müntz spaces as model spaces, to appear in Complex Analysis and Operator Theory.

[7] L. Gaillard, P. Lefèvre, Lacunary Müntz spaces: isomorphisms and Carleson embeddings, to appear in Ann. Inst. Fourier.

[8] L. Gaillard, P. Lefèvre, Asymptotic isometry for lacunary Müntz spaces and applications, preprint.

[9] G. Godefroy, Unconditionality in spaces of smooth functions, Archiv der Math. 92 (2009), 476-484.

[10] V.Gurariy and W. Lusky, Geometry of Müntz spaces and related questions, Lecture Notes in Mathematics, Springer, Berlin Heidelberg New York, (2005).

[11] V. Gurariy and V.I. Macaev, Lacunary power sequences in the spaces $C$ and $L^{p}$, Amer. Math. Soc. Translated, Serie 2, Vol.72, (1966), 9-21.

[12] D. Li and H. Queffélec, Introduction à l'étude des espaces de Banach - Analyse et probabilités, Cours Spécialisés 12, Société Mathématique de France (2004).

[13] S. Ludkowski, Approximation and existence of Schauder bases in Müntz spaces of $L^{1}$ functions, J. Math. Anal. Appl. 441 (2016), no. 2, 635-647.

[14] S. Ludkowski, Approximation in Müntz spaces $M_{\Lambda, p}$ of $L^{p}$ functions for $1<p<\infty$ and bases, arXiv:1605.09665v2.

[15] S. Ludkowski, Approximation and Schauder bases in Müntz spaces $M_{\Lambda, C}$ of continuous functions, arXiv:1605.09661v2.

[16] S. Ludkowski and W. Lusky, On the geometry of Müntz spaces, J. Funct. Spaces 2015, Article ID 787291.

[17] C. Müntz, Über den Approximationssatz von Weierstrass, H. A. Schwarz's Festschrift, Berlin, 1914, pp.303-312.

[18] S.W. Noor, D. Timotin, Embeddings of Mütz spaces: the Hilbertian case, Proc. Amer. Math. Soc. 141 (2013), no. 6, 2009-2023.

[19] W. Rudin, Real and complex analysis.

[20] O. Szász, Über die Approximation stetiger Funktionen durch lineare Aggregate von Potenzen, Math. Ann., 77 (1916), 482-496.

[21] D. Werner, A remark about Müntz spaces, http://page.mi.fu-berlin.de/werner/preprints /muentz.pdf.

Laboratoire de Mathématiques de Lens (LML), EA 2462, FÉdération CNRS NordPas-de-Calais FR 2956, Université D'Artois, Rue Jean Souvraz S.P. 18, 62307 Lens Cedex, France

E-mail address: pascal.lefevre@univ-artois.fr 